\title{
ESTIMACIÓN DE LAS PÉRDIDAS BIO-ECONÓMICAS CAUSADAS POR LA CAPTURA DE JUVENILES DE ANCHOVETA (Engraulis ringens, J.) EN LA COSTA PERUANA
}

\section{ESTIMATION OF BIO-ECONOMIC LOSSES CAUSED BY THE CAPTURE OF JUVENILE ANCHOVY (Engraulis ringens, J.) IN THE PERUVIAN COAST}

\author{
Renato Salvatteci ${ }^{1}$ y Jaime Mendo ${ }^{1}$
}

\begin{abstract}
Resumen
Durante muchos años los desembarques de la pesquería pelágica en la costa peruana se han caracterizado por la presencia de un alto porcentaje de individuos juveniles, especialmente en el caso de la anchoveta (Engraulis ringens) durante el año 2002 con consecuencias poco conocidas y estudiadas. En este trabajo se analizan las pérdidas en biomasa y económicas que la pesca de juveniles ocasiona basado en proyecciones de la composición por tallas de los desembarques de los puertos de Paita, Chimbote, Callao y Pisco. Las proyecciones de biomasa, se realizaron mediante el modelo predictivo de Thompson y Bell utilizando parámetros de crecimiento de von Bertalanffy estimados para cada puerto mediante el programa ELEFAN I y de la tasa de mortalidad natural proporcionado por el Instituto del Mar del Perú (IMARPE). Las biomasas fueron proyectadas hasta que la fracción de individuos $<12 \mathrm{~cm}$ presentara un porcentaje menor o igual al $10 \%$. Las pérdidas económicas fueron estimadas restando el valor de la biomasa proyectada y la captura. Los coeficientes de conversión, estimados usando información de la industria harinera, y los precios internacionales fueron usados para estimar la producción y el valor de la harina y aceite de pescado.

Los resultados muestran que al proyectar los desembarques reales las biomasas aumentaron en: $13.3 \%$ en Paita, $17.7 \%$ en Chimbote, $9.3 \%$ en Callao y $7.6 \%$ en Pisco y el valor de las biomasas proyectadas procesadas en harina y aceite aumentaron en $31.8 \%$ en Paita, $40.9 \%$ en Chimbote, $25.9 \%$ en Callao y $21.2 \%$ en Pisco. Relacionando el valor de la captura y la biomasa proyectada para el año 2002 se obtuvo una diferencia de 91.8 millones de dólares. Se discute el impacto de esta estrategia de pesca sobre la dinámica poblacional y se sugieren algunas medidas de manejo para incrementar el rendimiento biológico y económico de esta especie.
\end{abstract}

Palabras claves: anchoveta, Engraulis ringens, composición por tallas, desembarque, modelos predictivos, harina y aceite de pescado.

\begin{abstract}
During many years the landings of the pelagic fishery off the Peruvian coast have been characterized by a high percentage of juvenile individuals, especially in the case of the anchovy (Engraulis ringens) during the year 2002 whose impacts are not well-known and studied. In this work we analyzed the biomass and economic loss due to the fishing of juveniles based on projections of the size composition of the landings in Paita, Chimbote, Callao and Pisco. The biomass projections were carried out by means of the Thompson and Bell model using the von Bertalanffy growth parameters estimated with the program ELEFAN I for each landing site and the natural mortality rate provided by the Peruvian Marine Institute (IMARPE). The biomasses were projected until the $<12 \mathrm{~cm}$ fraction of individuals presented a percentage lesser than $10 \%$. The economic losses were estimated by subtracting the value of landing from the value of the projected biomass. Conversion coefficients were estimated using available data of the fish meal industry and international prices for fish meal and oil were used in order to estimate fish meal and oil production and value.

The results show that when projecting the real landings the biomasses increase by: $13.3 \%$ in Paita, $17.7 \%$ in Chimbote, $9.3 \%$ in Callao and $7.6 \%$ in Pisco and the value of the processed biomasses by $31.8 \%$ in Paita, $40.9 \%$ in Chimbote, $25.9 \%$ in Callao and $21.2 \%$ in Pisco. In relation to landing value and biomass projected for the year 2002 a difference of 91.8 million dollars was obtained. We discuss the impact of this fishing strategy on the population dynamics and some management measures are suggested to increase the biological and economic yield of this species.
\end{abstract}

Key words: anchoveta, Engraulis ringens, size composition, landing, predictive model, fish meal and oil. 


\section{Introducción}

Los recursos pesqueros frente a las costas del Pacífico Sur-oriental y de manera especial los recursos pelágicos, son muy abundantes y su disponibilidad está en relación directa con la intensidad y persistencia de los procesos de afloramiento, que hacen de esta área una de las más productivas del mundo (Ryther, 1969). En el Perú, la anchoveta (Engraulis ringens) es la principal especie capturada y es utilizada casi en su totalidad para la producción de harina y aceite de pescado. Por otro lado, debido a su posición central en el ecosistema, es considerada como una especie clave (Pauly, 1987 b; Pauly \& Tsukayama, 1987; Muck, 1989).

Es conocido que durante muchos años la pesquería ha incidido fuertemente sobre ejemplares menores de la talla mínima legal, especialmente durante el año 2002, donde los porcentajes de captura de juveniles son mayores que lo permitido legalmente $(<10 \%$ individuos menores de $12 \mathrm{~cm}$.). Ello podría significar el riesgo de una sobrepesca por crecimiento y reclutamiento de la anchoveta y con ello una reducción del beneficio económico de esta pesquería. Por otro lado se sabe que en el procesamiento de harina el rendimiento pescado - harina y por lo tanto la rentabilidad es mayor cuando se utiliza como materia prima individuos adultos, por lo que dejar crecer a los juveniles incrementa el rendimiento económico y además aumentaría la biomasa de los progenitores lo que podría significar un mejor reclutamiento en la población.

Un análisis exhaustivo de la estructura por tallas de los desembarques y como se vería afectada esta ante la aplicación de vedas, así como el posible impacto biológico y económico en la pesquería hasta ahora no se ha publicado. Schaefer (1967) e IMARPE (1970) sugiere que la protección de juveniles tendría dos efectos beneficiosos que se traducen en el incremento de la biomasa de progenitores y del rendimiento económico.

Es por ello que en el presente estudio se analiza en términos biológicos y económicos el impacto que ocasiona la captura de juveniles con la finalidad de dar algunas pautas de manejo que contribuyan a optimizar los beneficios que ofrece la explotación de la anchoveta en la costa norte peruana.

\section{Materiales y métodos}

Para la realización de este trabajo se utilizó la composición por tallas de los desembarques de la anchoveta peruana en el año 2002 provenientes de los puertos de Paita $\left(05^{\circ} 05^{\prime} \mathrm{S}\right.$ y $\left.81^{\circ} 07^{\prime} \mathrm{W}\right)$, Chimbote $\left(9^{\circ}\right.$ 4' $\left.15^{\prime \prime} \quad \mathrm{S}, \quad 78^{\circ} \quad 35^{\prime} \quad 27^{\prime \prime} \mathrm{W}\right)$, Callao $\left(12^{\circ} 02^{\prime} 42^{\prime}\right.$ 'S $\left.77^{\circ} 08^{\prime} 36^{\prime \prime} \mathrm{W}\right)$ y Pisco $\left(13^{\circ} 48^{\prime} 09^{\prime \prime} \mathrm{S} 76^{\circ} 17^{\prime} 23^{\prime \prime} \mathrm{W}\right)$, que tradicionalmente presentan los mayores desembarques en la costa peruana. Estos datos fueron proporcionados gentilmente por el Instituto del Mar del Perú (IMARPE).

Un esquema general del proceso metodológico seguido en el presente estudio se muestra en la Figura 1.

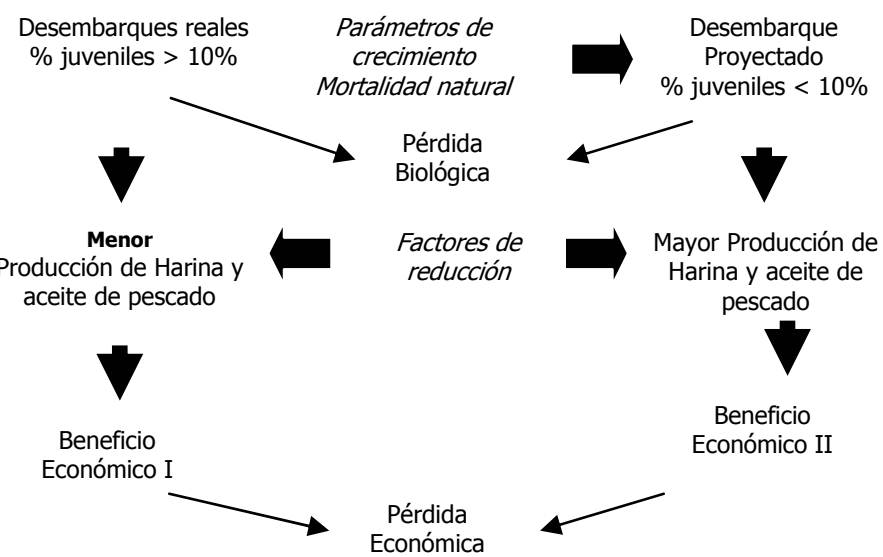

Figura 1. Esquema del proceso utilizado en ese trabajo para determinar el impacto biológico y económico de la captura de juveniles de anchoveta.

\section{Proyecciones de biomasa}

Las proyecciones en biomasa basadas en la composición por tallas de los desembarques de cada puerto fueron realizadas con el modelo predictivo de Thompson y Bell (Thompson \& Bell, 1934; Sparre \& Venema, 1997) hasta que el porcentaje de juveniles $(<12 \mathrm{~cm})$ en los desembarques sea menor o igual al $10 \%$. Para ello se estimaron los parámetros de crecimiento de la ecuación de von Bertalanffy:

$\mathrm{L}_{\mathrm{t}}=\mathrm{L}_{\infty} *\left[1-\exp ^{(-\mathrm{K} *(\mathrm{t}-\mathrm{to}))}\right]$

Donde: $\quad L_{t}=$ Longitud en un tiempo $t$

$\mathrm{L}_{\infty}=$ Longitud infinita

$\mathrm{K}=$ parámetro de curvatura

$\mathrm{t}=$ edad

$\mathrm{t}_{\mathrm{o}}=\mathrm{t}$-cero, edad a una longitud cero

Los parámetros de crecimiento de, $\mathrm{K}$ y Loo, usados en la proyección fueron estimados para cada puerto mediante el programa ELEFAN I (Electronic LEngth Frecuency ANalysis) incluido en el paquete computacional FISAT (FAO-ICLARM Stock Assessment Tools) (Pauly, 1987a). El valor de $t_{0}$ se obtuvo utilizando la ecuación de crecimiento inversa de von Bertalanffy $\mathrm{t}(\mathrm{L})=\mathrm{t}_{\mathrm{o}}-(1 / \mathrm{K}) * \ln (1-\mathrm{L} / \mathrm{Loo})$ y los valores de $\mathrm{t}(\mathrm{L})=21.9$ días y $\mathrm{L}=14.1 \mathrm{~mm}$ reportados por Palomares et al. (1987). La proyección del crecimiento se realizó con la ecuación inversa de von Bertalanffy y se reestructuró al intervalo de 0.5 $\mathrm{cm}$ inferior,

El número de sobrevivientes se estimó mediante la ecuación de extinción exponencial

$\mathrm{N}_{\mathrm{t}}=\mathrm{N}(\mathrm{t}-1) * \exp ^{\left[-\mathrm{Z}^{*}(\mathrm{t}-(\mathrm{t}-1)]\right.}$, 
Donde: $\quad \mathrm{N}_{\mathrm{t}}=$ Número de sobrevivientes $\mathrm{N}_{\mathrm{t}-1}=$ Número de sobrevivientes en un tiempo anterior

$\mathrm{t}-1=$ edad de los sobrevivientes $\mathrm{N}_{\mathrm{t}-1}$

$\mathrm{t}=$ edad de los sobrevivientes $\mathrm{N}_{\mathrm{t}}$

$\mathrm{Z} \quad=$ tasa de mortalidad total, $\mathrm{Z}=\mathrm{M}$

La tasa de mortalidad natural (M) utilizada para la estimación del número de sobrevivientes fue de $\mathrm{M}=0.8 / \mathrm{año}$, y la relación Peso-talla usada para convertir el número en biomasa fue $\mathrm{P}=0.00294 *$ $\mathrm{L}^{3.33}$. Ambos datos fueron proporcionados por IMARPE. El número de individuos en cada grupo de talla fue multiplicado por el peso promedio correspondiente y finalmente estas fueron sumadas para obtener la biomasa total o desembarque proyectado.

Las pérdidas en biomasa fueron estimadas por diferencia entre el volumen de desembarque real y la biomasa proyectada para cada mes y para cada puerto.

\section{Pérdidas económicas}

Las pérdidas económicas fueron estimadas restando el valor de la biomasa proyectada y el valor del desembarque real. La conversión pescado-harina y pescado-aceite fue realizada con datos de conversión correspondientes a los años 2001 y 2002 proporcionados por la empresa Austral, los cuales debieron ser modificados considerando solo la conversión de anchoveta sin desperdicios ni otras especies y la variación de la producción de harina y aceite en relación a la proporción de juveniles en el proceso. Finalmente, los precios internacionales de harina y aceite de pescado, vigentes para el año 2002 fueron tomados de Pesca (2003) para estimar el valor de la producción de harina y aceite de pescado.

\section{Resultados y discusión \\ Composición de la captura}

La Tabla 1 muestra la proporción y número de juveniles en la captura en los diferentes meses y puertos durante el 2002. En ella se puede apreciar que a excepción de Paita los únicos meses en los cuales se presentan altos porcentajes de juveniles son Enero, Marzo, Abril, Mayo y Junio. Los meses de Febrero, Agosto y Septiembre no presentan desembarques porque son meses de veda reproductiva.

\section{Parámetros de crecimiento}

La Tabla 2 muestra los valores de los parámetros de crecimiento de la ecuación de Von Bertalanffy estimados para la anchoveta peruana y para cada lugar de desembarque en el año 2002. Estos valores son similares a los parámetros reportados por diversos autores como Palomares et al. (1987); Mendo (1991); Pauly \& Tsukayama (1983); Aguayo (1976).

\section{Proyecciones en biomasa}

A manera de ejemplo la Fig. 2 muestra los resultados de la proyección de los desembarques de anchoveta en el puerto de Paita. La primera columna de cada proyección representa el desembarque real en toneladas y las restantes columnas las biomasas proyectadas mes a mes. Conforme los individuos van creciendo y el número disminuye por causas naturales la biomasa se incrementa. En algunos casos hubiese bastado 2 meses, como en la proyección del desembarque del mes de Mayo, para que la proporción de juveniles se reduzca a menos del $10 \%$ como exige la normatividad vigente. En otros como en el mes de Junio, se necesitaría hasta 3 meses para ello. El tiempo necesario para alcanzar un porcentaje de juveniles $<10 \%$ depende de las tallas iniciales de estos en los desembarque reales.

Tabla 1. Porcentaje de juveniles de anchoveta (Engraulis ringens) registrado en los desembarques de Paita, Chimbote, Callao y Pisco en el año 2002.

\begin{tabular}{lcccc}
\hline \multicolumn{1}{c}{ Mes } & Paita & Chimbote & Callao & Pisco \\
\hline Enero & 24.34 & 84.15 & 49.94 & 54.07 \\
Febrero & VEDA & VEDA & VEDA & VEDA \\
Marzo & 46.99 & 75.37 & 77.25 & 40.73 \\
Abril & 51.15 & 78.66 & 76.26 & 53.8 \\
Mayo & 41.57 & 43.12 & 34.24 & 25.1 \\
Junio & 49.2 & 13.41 & 14.17 & 8.57 \\
Julio & 33.03 & 3.23 & 0.33 & 12.87 \\
Agosto & VEDA & VEDA & VEDA & VEDA \\
Setiembre & VEDA & VEDA & VEDA & VEDA \\
Octubre & 0.38 & 0.06 & 0 & 0 \\
Noviembre & 0 & 1.15 & 0.01 & 0 \\
Diciembre & 0 & 0.19 & 0.01 & 0 \\
\hline
\end{tabular}

Tabla 2. Parámetros de crecimiento de la ecuación de Von Bertalanffy estimados para la anchoveta peruana (Engraulis ringens) en el año 2002 utilizando el programa ELEFAN I.

\begin{tabular}{cccc}
\hline PUERTO & $\mathbf{K}$ & $\mathbf{L}_{\mathbf{0 0}}$ & $\mathbf{t}_{\mathbf{0}}$ \\
\hline Paita & 0.99 & 19.9 & -0.014 \\
Chimbote & 1.00 & 19.0 & -0.017 \\
Callao & 0.85 & 19.4 & -0.029 \\
Pisco & 1.00 & 19.1 & -0.017 \\
\hline Promedio & $\mathbf{0 . 9 6}$ & $\mathbf{1 9 . 3 5}$ & $\mathbf{- 0 . 0 1 9 3}$ \\
\hline
\end{tabular}

La ganancia en biomasa obtenida de las proyecciones de los desembarques se muestra para cada puerto en la Tabla 3. Los valores negativos se presentan cuando las pérdidas por mortalidad natural superan la ganancia por crecimiento somático. Las proyecciones arrojan una ganancia total aproximada de 255993 t en biomasa (13.2\%) en los 4 puertos, $y$ las más altas corresponden al puerto de Chimbote en 
donde la ganancia llega a las 154 mil toneladas que representa un incremento de $17.7 \%$ en relación a los desembarques reales.

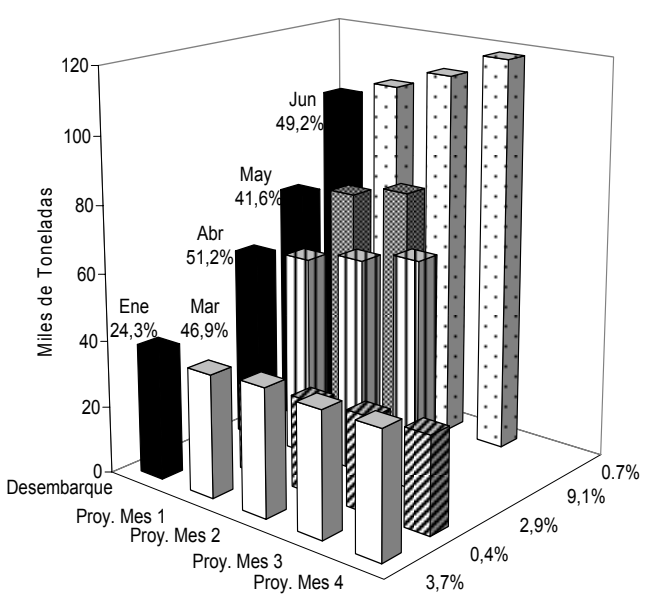

Figura 2. Proyecciones en biomasa (miles de toneladas) de anchoveta en el puerto de Paita desde Enero a Junio del 2002. Los porcentajes indican la proporción de juveniles en los desembarques reales (columnas negras) y en la biomasa al final de la proyección

El número de meses proyectado para alcanzar una proporción de juveniles menor de $10 \%$ en la composición por tallas de la biomasa varía entre 1 y 5 meses. Este número esta relacionado directamente al tamaño promedio de la fracción de juveniles en los desembarques, tal como es el caso de los desembarques de Junio en Chimbote, Callao y Pisco. Contrariamente, para el desembarque del mes de Enero en Chimbote fue necesario una proyección de 5 meses para alcanzar que la fracción de individuos $<12 \mathrm{~cm}$ sea $<10 \%$.

Las ganancias en biomasa podrían tener otros beneficios relacionados con el potencial reproductivo y el reclutamiento así como en términos ecotróficos. Una veda dependiente de la talla de los juveniles especialmente en el verano/otoño significaría una mayor cantidad de adultos y por lo tanto una liberación de productos sexuales y un mayor reclutamiento. El número total de individuos que pasan de juveniles a adultos según las proyecciones realizadas (Tabla 4) fue 60597 millones de individuos. Considerando una proporción sexual de hembras y machos de 1:1 y la fecundidad de las hembras de 15000 ovocitos (Miñano, 1968; Santander et al., 1984), el número de ovocitos que se habrían dejado de liberar fue de $454 \times 10^{12}$. De esta manera los reclutamientos para el año 2003 podrían haberse afectado, tal como lo sugiere la disminución de los volúmenes de desembarque de 8.1 millones de $\mathrm{t}$ en el 2002 a 5.1 millones de t en el 2003.
Tabla 3. Desembarques (miles de $\mathrm{t}$ ) de anchoveta y biomasa proyectada (miles de t) en los puertos de Paita, Chimbote, Callao y Pisco durante el 2002.

\begin{tabular}{|c|c|c|c|c|c|c|c|}
\hline \multirow[b]{2}{*}{ PUERTO } & \multicolumn{2}{|c|}{ Desembarques } & \multicolumn{3}{|c|}{ Biomasa Proyectada } & \multicolumn{2}{|c|}{ Incremento } \\
\hline & Mes & $\begin{array}{c}\text { miles } \\
\text { de t }\end{array}$ & Mes & $\begin{array}{c}\text { miles } \\
\text { de t }\end{array}$ & Meses & $\begin{array}{c}\text { miles } \\
\text { de t }\end{array}$ & $\%$ \\
\hline \multirow{6}{*}{ PAITA } & Enero & 39 & Mayo & 37 & 4 & -1 & -3.48 \\
\hline & Marzo & 26 & Julio & 29 & 4 & 3 & 12.47 \\
\hline & Abril & 57 & Julio & 68 & 3 & 11 & 18.77 \\
\hline & Mayo & 72 & Julio & 79 & 2 & 7 & 10.15 \\
\hline & Junio & 99 & Septiem & 118 & 3 & 19 & 19.2 \\
\hline & Subtotal & 292 & & 331 & & 39 & 13.3 \\
\hline \multirow{6}{*}{ CHIMBOTE } & Enero & 108 & Junio & 147 & 5 & 40 & 37.19 \\
\hline & Marzo & 91 & Julio & 119 & 4 & 28 & 31.34 \\
\hline & Abril & 182 & Agosto & 239 & 4 & 57 & 31.59 \\
\hline & Mayo & 282 & Agosto & 318 & 3 & 35 & 12.4 \\
\hline & Junio & 205 & Julio & 198 & 1 & -7 & -3.54 \\
\hline & Subtotal & 868 & & 1021 & & 154 & 17.7 \\
\hline \multirow{6}{*}{ CALLAO } & Enero & 0 & Abril & 0 & 3 & 0 & 4.52 \\
\hline & Marzo & 37 & Julio & 46 & 4 & 9 & 23.42 \\
\hline & Abril & 66 & Agosto & 81 & 4 & 15 & 22.55 \\
\hline & Mayo & 103 & Julio & 107 & 2 & 4 & 3.60 \\
\hline & Junio & 63 & Julio & 60 & 1 & -2 & -3.47 \\
\hline & Subtotal & 269 & & 294 & & 25 & 9.3 \\
\hline \multirow{5}{*}{ PISCO } & Enero & 6 & Abril & 7 & 3 & 1 & 13.85 \\
\hline & Marzo & 120 & Junio & 134 & 3 & 14 & 11.66 \\
\hline & Abril & 141 & Julio & 163 & 3 & 22 & 15.88 \\
\hline & Mayo & 238 & Junio & 240 & 1 & 1 & 0.49 \\
\hline & Subtotal & 505 & & 544 & & 38 & 7.6 \\
\hline TOTAL & & 1935 & & 2191 & & 256 & 13.2 \\
\hline
\end{tabular}

Según varios autores (IMARPE, 1973, 1974; Pauly \& Tsukayama, 1987; Csirke et al., 1996; Csirke \& Gummy, 1996) la fuerte presión de pesca a la que fue sometida la población de anchoveta a finales de los sesenta e inicios de los setentas, además de El Niño de 1972, fueron causas principales para el colapso de esta pesquería. Sin embargo, no hay que olvidar que, antes del colapso de la pesquería, los desembarques de anchoveta mostraron un alto porcentaje de juveniles tal como en el año 2002 (Tabla 5), llegándose a capturar de Enero a Mayo 4.2 millones de t en 1970 (62.2\%) y 2 millones de toneladas en 1971 (45.0\%).

Por otro lado buena parte de la mortalidad natural durante todo el tiempo de proyección iría a parar como alimento de otras especies como el jurel, la caballa, el bonito y la merluza entre otras. A mediano o largo plazo ello beneficiaria a estas poblaciones y por lo tanto a sus pesquerías. Muck (1989) al respecto sostiene que la anchoveta es el eje en el que interactúan muchas especies claves en el ecosistema pelágico frente al Perú por lo que del aumento o 
disminución de la biomasa de anchoveta dependerán el resto de especies.

Finalmente es importante citar a Froese (2004) quien simplifica el manejo de una especie en solo tres frases: "dejémos desovar a la especie", "dejémos crecer a la especie" y "dejemos vivir a los megadesovadores".

Tabla 4. Número de individuos juveniles (en millones) en los desembarques por puerto que llegaron a tallas mayores de $12 \mathrm{~cm}$ luego de la proyección realizada hasta alcanzar $<10 \%$ de juveniles.

\begin{tabular}{cccccc}
\hline PUERTO & A & B & C & D & E \\
\hline PAITA & 10245 & 2167 & 8079 & 218 & 7860 \\
CHIMBOTE & 45845 & 10443 & 35402 & 2017 & 33385 \\
CALLAO & 11973 & 2385 & 9588 & 1485 & 8103 \\
PISCO & 15271 & 2262 & 13009 & 1760 & 11248 \\
\hline TOTAL & $\mathbf{8 3 3 3 5}$ & $\mathbf{1 7 2 5 7}$ & $\mathbf{6 6 0 7 8}$ & $\mathbf{5 4 8 1}$ & $\mathbf{6 0 5 9 7}$ \\
\hline
\end{tabular}

$\mathrm{A}=$ Número de juveniles en los desembarques; $\mathrm{B}=$ Número de individuos muertos en la proyección; $\mathrm{C}=$ Número de sobrevivientes; $\mathrm{D}=$ Número de juveniles en la proyección; $\mathrm{E}=$ Número de juveniles que alcanzaron tallas $>12 \mathrm{~cm}$.

Tabla 5. Desembarques (millones de $t$ ) de anchoveta y porcentaje de juveniles durante los 5 primeros meses del año entre 1968 - 1970

\begin{tabular}{|c|c|c|c|c|}
\hline \multirow[t]{2}{*}{ Año } & \multirow[t]{2}{*}{ Mes } & \multirow{2}{*}{$\begin{array}{c}\text { Desembarque } \\
\text { (mill. de t) }\end{array}$} & \multicolumn{2}{|c|}{ Juveniles } \\
\hline & & & mill. $t$ & $\%$ \\
\hline \multirow{6}{*}{1968} & Enero & 1.47 & 0.8 & 55.5 \\
\hline & Febrero & 0.9 & 0.6 & 67.8 \\
\hline & Marzo & 0.74 & 0.5 & 70.3 \\
\hline & Abril & 1.08 & 0.4 & 34.2 \\
\hline & Mayo & 0.95 & 0.4 & 45.6 \\
\hline & TOTAL & 5.14 & 2.7 & 53.5 \\
\hline \multirow{6}{*}{1969} & Enero & 1.23 & 1.1 & 87.1 \\
\hline & Febrero & - & - & - \\
\hline & Marzo & 1.76 & 1.4 & 78.6 \\
\hline & Abril & 1.25 & 0.4 & 30.4 \\
\hline & Mayo & 0.79 & 0.3 & 41.9 \\
\hline & TOTAL & 5.03 & 3.2 & 62.9 \\
\hline \multirow{6}{*}{1970} & Enero & 2 & 1.7 & 86.5 \\
\hline & Febrero & 0.99 & 0.7 & 67.7 \\
\hline & Marzo & 1 & 0.4 & 43.4 \\
\hline & Abril & 1.9 & 1.0 & 55.0 \\
\hline & Mayo & 0.83 & 0.3 & 36.6 \\
\hline & TOTAL & 6.72 & 4.2 & 62.2 \\
\hline \multirow{6}{*}{1971} & Enero & - & - & - \\
\hline & Febrero & - & - & - \\
\hline & Marzo & 2.37 & 1.1 & 45.1 \\
\hline & Abril & 1.45 & 0.7 & 48.2 \\
\hline & Mayo & 0.64 & 0.2 & 37.1 \\
\hline & TOTAL & 4.46 & 2.0 & 45.0 \\
\hline TOTAL & & 21.35 & & \\
\hline
\end{tabular}

\section{Pérdidas económicas}

Las relaciones obtenidas entre el porcentaje de juveniles en los desembarques y el coeficiente de conversión pescado-harina y pescado-aceite presentan un buen ajuste (Fig. 3 y 4).

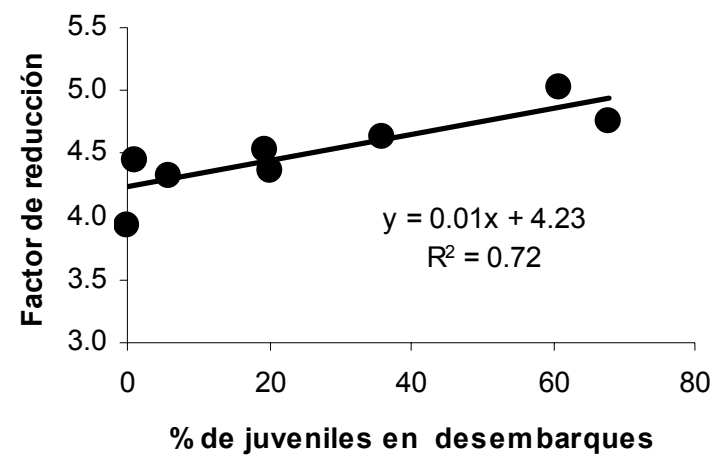

Figura 3. Relación entre el porcentaje de juveniles en los desembarques de anchoveta $y$ el factor de reducción de pescado-harina.

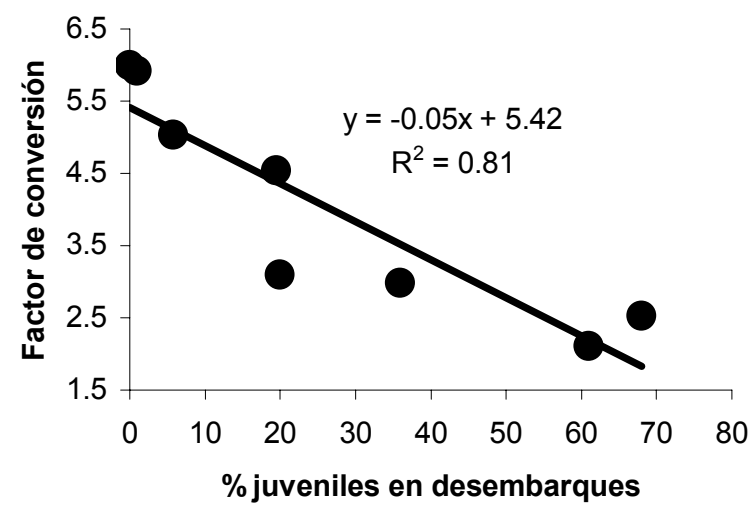

Figura 4. Relación entre el porcentaje de juveniles en los desembarques de anchoveta $y$ el factor de conversión de pescado-aceite.

La Tabla 6 muestra los volúmenes de harina producidos a partir de los desembarques y la biomasa proyectada, en la cual se puede observar un aumento de 413.4 a 512.7 miles de t, es decir un incremento de 99.4 miles de t (24\%). En cuanto a la producción de aceite, esta aumenta de 60.3 a 114.1 miles de t, lo cual significa un incremento de 53.8 miles de t $(89.2 \%)$, el cual se explica por el bajo contenido de grasa de los individuos juveniles con relación a los adultos. Los mayores incrementos se observan en el puerto de Chimbote en donde la harina de pescado producida a partir de los desembarques se incrementa de $183.3 \mathrm{mil}$ toneladas a 239.6 mil toneladas luego de la proyección, lo que representa un incremento de $30.8 \%$. En el caso del aceite de pescado la producción se incrementa de 24.5 a 53.7 mil toneladas lo que representa un incremento de $119.5 \%$. 
Tabla 6. Producción (miles de t) de harina y aceite de pescado estimada a partir de los desembarques y la biomasa proyectada para los puertos de Paita, Chimbote, Callao y Pisco durante el 2002.

\begin{tabular}{|c|c|c|c|c|c|c|c|c|c|c|}
\hline \multirow[t]{2}{*}{ PUERTO } & \multicolumn{3}{|c|}{$\begin{array}{l}\text { Producción desembarques } \\
\text { (miles de t) }\end{array}$} & \multicolumn{3}{|c|}{$\begin{array}{c}\text { Producción biomasa } \\
\text { proyectada } \\
\text { (miles de } t) \\
\end{array}$} & \multicolumn{4}{|c|}{$\begin{array}{l}\text { Incremento } \\
\text { (miles de t) }\end{array}$} \\
\hline & Mes & Harina & Aceite & Mes & Harina & Aceite & Harina & $\%$ & Aceite & $\%$ \\
\hline \multirow{6}{*}{ PAITA } & Enero & 8.6 & 1.6 & Mayo & 8.8 & 2.0 & 0.1 & 1.2 & 0.3 & 20.2 \\
\hline & Marzo & 5.5 & 0.8 & Julio & 6.8 & 1.6 & 1.4 & 24.8 & 0.8 & 97.5 \\
\hline & Abril & 12.0 & 1.6 & Julio & 15.9 & 3.6 & 3.9 & 32.2 & 1.9 & 118.9 \\
\hline & Mayo & 15.5 & 2.4 & Julio & 18.3 & 3.9 & 2.9 & 18.4 & 1.5 & 63.7 \\
\hline & Junio & 21.0 & 2.9 & Septiembre & 27.9 & 6.4 & 6.9 & 32.9 & 3.4 & 116.9 \\
\hline & Subtotal & 62.6 & 9.4 & Subtotal & 77.7 & 17.4 & 15.1 & 24.1 & 8.0 & 85.2 \\
\hline \multirow{6}{*}{ CHIMBOTE } & Enero & 21.2 & 1.3 & Junio & 34.7 & 7.9 & 13.5 & 63.9 & 6.6 & 504.7 \\
\hline & Marzo & 18.2 & 1.5 & Julio & 27.8 & 6.1 & 9.6 & 52.8 & 4.6 & 309.7 \\
\hline & Abril & 36.2 & 2.7 & Agosto & 56.3 & 12.8 & 20.1 & 55.5 & 10.1 & 372.7 \\
\hline & Mayo & 60.6 & 9.2 & Agosto & 74.6 & 16.8 & 14.0 & 23.2 & 7.6 & 82.5 \\
\hline & Junio & 47.0 & 9.7 & Julio & 46.1 & 10.1 & -0.9 & -2.0 & 0.3 & 3.4 \\
\hline & Subtotal & 183.3 & 24.5 & Subtotal & 239.6 & 53.7 & 56.4 & 30.8 & 29.2 & 119.5 \\
\hline \multirow{6}{*}{ CALLAO } & Enero & 0.0 & 0.0 & Abril & 0.0 & 0.0 & 0.0 & 18.8 & 0.0 & 100.0 \\
\hline & Marzo & 7.4 & 0.6 & Julio & 10.6 & 2.3 & 3.2 & 43.0 & 1.7 & 295.0 \\
\hline & Abril & 13.2 & 1.1 & Agosto & 18.8 & 4.1 & 5.6 & 42.1 & 3.0 & 284.3 \\
\hline & Mayo & 22.6 & 3.8 & Julio & 24.9 & 5.4 & 2.3 & 10.1 & 1.6 & 41.3 \\
\hline & Junio & 14.3 & 3.0 & Julio & 14.1 & 3.1 & -0.3 & -1.8 & 0.1 & 4.1 \\
\hline & Subtotal & 57.6 & 8.4 & Subtotal & 68.4 & 14.9 & 10.8 & 18.7 & 6.4 & 76.3 \\
\hline \multirow{5}{*}{ PISCO } & Enero & 1.3 & 0.2 & Abril & 1.7 & 0.4 & 0.4 & 28.2 & 0.2 & 126.3 \\
\hline & Marzo & 25.8 & 4.1 & Junio & 31.4 & 7.1 & 5.6 & 21.6 & 3.0 & 74.1 \\
\hline & Abril & 29.5 & 3.8 & Julio & 38.3 & 8.7 & 8.8 & 30.0 & 4.8 & 125.6 \\
\hline & Mayo & 53.2 & 9.9 & Junio & 55.6 & 12.0 & 2.4 & 4.4 & 2.1 & 20.9 \\
\hline & Subtotal & 109.9 & 18.0 & Subtotal & 127.0 & 28.1 & 17.1 & 15.6 & 10.1 & 56.2 \\
\hline TOTAL & & 413.4 & 60.3 & & 512.7 & 114.1 & 99.4 & 24.0 & 53.8 & 89.2 \\
\hline
\end{tabular}

En términos económicos el incremento total significa un ingreso adicional de 91.8 millones de dólares considerando los precios de harina y aceite de pescado vigentes en el año 2002 (Tabla 7). El mayor beneficio económico no solo se da por el aumento significativo de la biomasa proyectada, sino también por el mayor valor de la captura de individuos con tallas mayores destinados a la elaboración de harina y aceite de pescado.

Los resultados de este estudio nos llevan a analizar algunas medidas de manejo que podrían ser consideradas para evitar estas pérdidas o para obtener una mayor ganancia sin dañar a la población de anchoveta y a aquellos que dependen de ella. Es claro que una veda de 2 o 3 meses afectaría a la flota pesquera, ya que durante este tiempo las empresas dejarían de percibir ingresos, sin embargo el mayor ingreso que se percibiría por procesar anchoveta adulta podría ser destinado a aliviar los costos que surgirían ante una paralización de la flota o frente a los mayores costos de operación en épocas con mayor dispersión del recurso. Por otro lado, la pesca de juveniles mayormente se registra cerca de la costa donde habitan otras especies que son aprovechadas por la pesca artesanal y que son también extraídas por la flota anchovetera. Ello reduciría enormemente los constantes conflictos con la pesca artesanal.

La suspensión de la pesquería solo se debe aplicar en los puertos o zonas de pesca donde se observan abundancia de juveniles, lo que no prohibiría pescar en otras zonas con presencia de individuos adultos. El uso de un modelo predictivo basado en los desembarques de los diferentes puertos, tal como ha sido aplicado en este estudio, proporcionaría la magnitud de la paralización de la pesca que está relacionada con los tamaños y los porcentajes de juveniles.

Aun cuando la normatividad vigente permite solo un $10 \%$ de juveniles en los desembarques, en la práctica esta norma no se aplica rigurosamente. La pesca 
indiscriminada de juveniles merece ser analizada considerando las pérdidas biológicas y económicas mostradas en el presente estudio con la finalidad de cumplir fielmente con los principios de pesca responsable.

Tabla 7. Valor de la harina y aceite de pescado en millones de dólares estimado a partir de los desembarques (A) y de la biomasa proyectada (B) para los puertos de Paita, Chimbote, Callao y Pisco durante el 2002.

\begin{tabular}{|c|c|c|c|c|c|c|}
\hline \multirow[t]{2}{*}{ PUERTO } & \multicolumn{2}{|c|}{ A } & \multicolumn{2}{|c|}{ B } & \multicolumn{2}{|c|}{$\begin{array}{c}\text { Incremento } \\
\text { (B-A) }\end{array}$} \\
\hline & Mes & mill. US\$ & Mes & mill. US\$ & mill. US\$ & $\%$ \\
\hline \multirow{6}{*}{ PAITA } & Enero & 6.2 & Mayo & 6.5 & 0.3 & 4.1 \\
\hline & Marzo & 3.8 & Julio & 5.1 & 1.3 & 33.8 \\
\hline & Abril & 8.2 & Julio & 11.7 & 3.5 & 42.3 \\
\hline & Mayo & 10.8 & Julio & 13.4 & 2.6 & 24.3 \\
\hline & Junio & 14.4 & Septiembre & 20.6 & 7.4 & 51.5 \\
\hline & Subtotal & 43.4 & Subtotal & 57.3 & 15.1 & 34.7 \\
\hline \multirow{6}{*}{ CHIMBOTE } & Enero & 13.6 & Junio & 25.7 & 12.1 & 88.7 \\
\hline & Marzo & 11.9 & Julio & 20.5 & 8.6 & 71.8 \\
\hline & Abril & 23.5 & Agosto & 41.6 & 18.1 & 76.9 \\
\hline & Mayo & 42.1 & Agosto & 55.1 & 13.0 & 30.8 \\
\hline & Junio & 34.2 & Julio & 33.8 & -0.4 & -1.1 \\
\hline & \begin{tabular}{|l|} 
Subtotal \\
\end{tabular} & 125.4 & Subtotal & 176.7 & 51.3 & 40.9 \\
\hline \multirow{6}{*}{ CALLAO } & Enero & 0.0 & Abril & 0.0 & 0.0 & 25.5 \\
\hline & Marzo & 4.8 & Julio & 7.8 & 2.9 & 60.7 \\
\hline & Abril & 8.6 & Agosto & 13.8 & 5.1 & 59.5 \\
\hline & Mayo & 16.0 & Julio & 18.3 & 2.3 & 14.5 \\
\hline & Junio & 10.4 & Julio & 10.3 & -0.1 & -0.8 \\
\hline & \begin{tabular}{|l|} 
Subtotal \\
\end{tabular} & 39.9 & Subtotal & 50.2 & 10.3 & 25.9 \\
\hline \multirow{5}{*}{ PISCO } & Enero & 0.9 & Abril & 1.3 & 0.4 & 39.1 \\
\hline & Marzo & 18.0 & Junio & 23.2 & 5.1 & 28.5 \\
\hline & Abril & 20.1 & Julio & 28.3 & 8.2 & 40.7 \\
\hline & Mayo & 38.1 & Junio & 40.7 & 2.7 & 7.0 \\
\hline & \begin{tabular}{|l|} 
Subtotal \\
\end{tabular} & 77.1 & Subtotal & 93.5 & 16.3 & 21.2 \\
\hline TOTAL & \multicolumn{3}{|c|}{285.9} & 377.7 & 91.8 & 32.1 \\
\hline
\end{tabular}

\section{Conclusiones}

- La biomasa proyectada tuvo un incremento de 256 mil t con respecto a los desembarques reales.

- La harina producida por los desembarques reales fue de 413.4 mil $\mathrm{t}$ frente a 512.7 mil t producidas por la biomasa proyectada, es decir, se obtuvo un incremento de 99.4 mil t (24\%).

- El aceite de pescado producido por los desembarques reales fue de 60.3 mil $t$ frente a 114.1 mil $t$ producido por la biomasa proyectada, es decir, se obtuvo un incremento de $53.8 \mathrm{mil} \mathrm{t}$ $(89.2 \%)$.

- El incremento del valor de la biomasa proyectada en relación al de los desembarques reales fue de 91.8 millones de dólares.

- La biomasa se incrementó en un $13.2 \%$ y las ganancias en un $32.1 \%$, lo que muestra claramente el mayor aporte de los individuos adultos en la producción de harina y aceite de pescado en relación a los juveniles.

\section{Agradecimientos}

Nuestro agradecimiento al Blgo. Miguel Ñiquen del Instituto del Mar del Perú (IMARPE) por la información proporcionada y por sus valiosas sugerencias en el desarrollo de este estudio, así como a la Dra. Claudia Wosnitza-Mendo por la revisión de una versión preliminar. Este estudio fue financiado y desarrollado en el marco del proyecto CENSOR de la Union Europea (Climate Variability and El Niño Southern Oscillation: implications for natural coastal resources and management, contrato 511071) y es la publicación Nro. 0023 de CENSOR.

\section{Literatura citada}

Aguayo H.M. 1976. Edad y crecimiento de la anchoveta (Engraulis ringens, Jenyns) del norte de Chile (Arica-Iquique). Invest. Pesq., Santiago 23 (1): 25

Csirke J. \& Gumy A. 1996. Análisis bioeconómico de la pesquería pelágica peruana dedicada a la producción de harina y aceite de pescado. Callao. Bol. Inst. Mar Perú - Callao.

Csirke J., Guevara-Carrasco R., Cárdenas G., Ñiquen M. \& Chipollini A. 1996. Situación de los recursos anchoveta (Engraulis ringens) y sardina (Sardinops sagax) a principios de 1994 y perspectivas para la pesca en el Perú, con particular referencia a las regiones norte y centro de la costa peruana. Callao, 3 pp. Bol. Inst. Mar Perú - Callao. Froese R. 2004. Keep it simple: three indicators to deal with overfishing. Fish and Fisheries. 5: 86-91. IMARPE. 1970. Panel de expertos informes sobre los efectos económicos de diferentes medidas regulatorias de la pesquería de la anchoveta peruana. Callao, 20 pp. Inf. Inst. Mar Perú-Callao. 1973. Tercera sesión del panel de expertos sobre la dinámica de la población de anchoveta peruana. Bol. Inst. Mar Perú - Callao. : 521-599

1974. Segundo panel de expertos. Informe sobre los efectos económicos de diferentes medidas regulatorias de la pesquería de la anchoveta peruana. Callao. Bol. Inst. Mar Perú - Callao.

Mendo J. 1991. Stock identification of Peruvian anchoveta (Engraulis ringens): morphometric, tagging/recapture, electrophoretic and ecological studies. Ph D. Thesis. Univ. Bremen, Alemania.

Miñano J.B. 1968. Estudio de la fecundidad y ciclo sexual de la anchoveta (Engraulis ringens $\mathrm{J}$.) en la zona de Chimbote. Bol. Inst. Mar del Peru-Callao. 1 (9).

Muck P. 1989. Major trends in the upwelling system off Peru and their implications for management, p. 386-403. In D. Pauly, P. Muck, J. Mendo and I. Tsukayama (eds.) The Peruvian upwelling ecosystem: dynamics and interactions. ICLARM Conference Proceedings 18, 438 p. Instituto del Mar del Perú (IMARPE), Callao, Perú; Deutsche Gesellschaft für Technische Zusammenarbeit (GTZ), GMBH, Eschborn, Federal Republic of Germany; and International Center for living Aquatic 
Resources Management (ICLARM), Manila, Philippines.

Palomares M.L., Muck P., Mendo J., Chumán de Flores E., Gómez O. \& Pauly D.. 1987. Growth of the Peruvian anchoveta (Engraulis ringens), 1953 to 1982, p. 117 141. In D. Pauly and I. Tsukayama (eds.) The Peruvian anchoveta and its upwelling ecosystem: three decades of change. ICLARM Studies and Reviews 15, $351 \mathrm{p}$, Instituto del Mar del Perú (IMARPE), Callao, Perú; Deutsche Gesellschaft für Technische Zusammenarbeit (GTZ), GMBH, Eschborn, Federal Republic of Germany; and International Center for Living Aquatic Resources Management (ICLARM), Manila, Philippines.

Pauly D. 1987 a. A review of the ELEFAN system for analysis of length-frequency data in fish and aquatic invertebrates. ICLARM Conf. Proc. (13) : 7-34.

1987 b. Managing the Peruvian upwelling ecosystem: a synthesis, p. $325-342$. In D. Pauly and I. Tsukayama (eds.) The Peruvian anchoveta and its upwelling ecosystem: three decades of change. ICLARM Studies and Reviews. 15: 351. Instituto del Mar del Perú (IMARPE), Callao, Perú; Deutsche Gesellschaft für Technische Zusammenarbeit (GTZ), GMBH, Eschborn, Federal Republic of Germany; and International Center for Living Aquatic Resources Management (ICLARM), Manila, Philippines.

Pauly D. \& Tsukayama I.. 1983. On the seasonal growth, monthly recruitment and monthly biomass of Peruvian anchoveta (Engraulis ringens) from 1961 to 1979, p. 987 - 1004. In: S. Sharp and J. Csirke (eds.) Proceedings of the Expert Consulation to Examine Changes in Abundance and Species Composition of Neritic Fish Resources. FAO Fish. Rep. 3 (291).
1987. On the implementation of management - oriented fishery research: the case of the Peruvian anchoveta, p.1 - 13. In D. Pauly and I. Tsukayama (eds.) The Peruvian anchoveta and its upwelling ecosystem: three decades of change. ICLARM Studies and Reviews 15, 351 p, Instituto del Mar del Perú (IMARPE), Callao, Perú; Deutsche Gesellschaft für Technische Zusammenarbeit (GTZ), GMBH, Eschborn, Federal Republic of Germany; and International Center for Living Aquatic Resources Management (ICLARM), Manila, Philippines.

Pesca. 2003. Revista de circulación mundial. Marzo - Abril 2003.

Ryther J.H. 1969. Photosynthesis and fish production in the sea. Science, 166:72-76.

Santander, H., J. Alheit y P.E. Smith. 1984. Estimación de la biomasa de la población desovante de anchoveta peruana Engraulis ringens en 1981 por aplicación del "Método de Producción de Huevos". Bol. Inst. Mar Perú Callao. 8 (6): 209-250.

Schaefer M.B. 1967.Dinámica de la pesquería de la anchoveta (Engraulis ringens), en el Perú. Callao, pp. 189-304. Bol. Inst. Mar Perú - Callao.

Sparre P. \&. Venema S.C. 1997. Introducción a la evaluación de recursos pesqueros tropicales. Parte I. Manual. FAO Documento Técnico de Pesca. No 306.1 Rev. 2.

Thompson W.F. \& Bell. F.H. 1934. Biological statistics of the Pacific halibut fishery. 2. Effect of changes in intensity upon total yield per unit of gear. Rep. Int. Fish. (Pacific halibut) Comm., (8): 49.

${ }^{1}$ Facultad de Pesquería, Universidad Nacional Agraria La Molina, Apartado postal 12-056, Lima 12, Perú 